
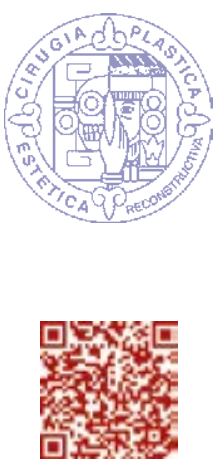

\title{
Manejo del seroma crónico en pacientes con implantes de mama en relación con el diagnóstico de linfoma anaplásico de células gigantes
}

\author{
Management of chronic seroma in patients with breast implants in \\ relation to the diagnosis of anaplastic lymphoma of giant cells \\ Dr. Guillermo Ramos-Gallardo,* Dr. Rodolfo Luciano Ríos-Lara y López, ${ }^{\ddagger}$ \\ Dr. Jesús Cuenca-Pardo,* Dr. Lázaro Cárdenas-Camarena,* Dra. Livia Contreras-Bulnes,* \\ Dra. Estela Vélez Benítez,* Dr. Javier Bucio-Duarte,* Dr. Mario Alberto Pineda-Espinosa, ${ }^{\ddagger}$ \\ Dr. Antonio Ugalde Vitelly, ${ }^{\ddagger}$ Dr. Héctor Manuel Avendaño-Peza ${ }^{\ddagger}$
}

\footnotetext{
Palabras clave: Linfoma anaplásico de células gigantes, BIA-ALCL, implantes mamarios, seroma crónico, seroma, linfoma asociado con implantes.

Keywords: Giant cell anaplastic lymphoma, BIA-ALCL, breast implants, chronic seroma, seroma, implantassociated lymphoma.
}

${ }^{*}$ Comité de Seguridad de la Asociación Mexicana de Cirugía Plástica, Estética y Reconstructiva, A.C.

¥ Servicio de

Cirugía Plástica y

Reconstructiva. Hospital General de México «Dr. Eduardo Liceaga».

Los autores de este artículo no tienen conflicto de intereses que declarar.

Recibido:

24 enero 2019

Aceptado para publicar:

04 marzo 2019

\section{RESUMEN}

En los últimos años hemos tenido mayor conocimiento de una enfermedad poco frecuente y relacionada con los implantes mamarios, se trata del linfoma anaplásico de células gigantes. Aunque son pocos los casos y la incidencia es variable, queremos mencionar lo que hemos hecho en nuestro país al respecto y las medidas que sugerimos para atender un posible caso. Es importante mencionar que no todos los casos de seroma crónico se han relacionado con linfoma, por lo que exponemos lo que se debe hacer ante un caso sospechoso, cómo manejar un seroma cuando el diagnóstico es negativo para BIA-ALCL (breast implant associated-anaplastic large cell lymphoma, por sus siglas en inglés) y recomendar que en este momento no hay indicación de retirar o cambiar implantes en pacientes asintomáticas. Al final — como anexo a este artículo- se agrega la infografía sobre el tema, hechas por el Comité de Seguridad para orientar a los cirujanos plásticos, personal de salud y pacientes.

\section{ABSTRACT}

In recent years, we have acquired more knowledge about a rare disease related to breast implants: the giant anaplastic lymphoma. Although there are few cases, and the incidence is variable, we want to mention what we have done in our country in this regard and the measures we suggest to handle a possible case. It is important to mention that not all cases of chronic seroma have been related to lymphoma. Therefore, we explain what should be done in a suspected case, how to handle a seroma when the diagnosis is negative for BIA-ALCL and recommend that implants should not be removed or changed in asymptomatic patients, at that time. Finally, as an annex to this article, the infographic on the subject made by the Safety Committee are added to guide plastic surgeons, health personnel and patients.

\section{INTRODUCCIÓN}

T os primeros registros en la literatura sobre ـel linfoma anaplásico de células gigantes se tienen en la década de los noventa. En la revista de nuestra especialidad, el primer caso aparece reportado en el año de 1997. ${ }^{1,2}$ Desde entonces hemos visto un incremento en la incidencia, junto con un mayor reporte y conocimiento de esta patología.
En 2016, revisamos la literatura y encontramos 80 casos. $^{3}$ De acuerdo con el Global Task Force contamos actualmente con más de 600 casos; posiblemente esta cifra aumente, por lo que debemos tener mayor conocimiento del problema. Esta patología ha causado un alto impacto en las autoridades sanitarias, sociedades médicas, medios de comunicación y pacientes. Además, la información que se genera con frecuencia es controvertida y no 
consistente. Aunque los casos que tenemos en nuestro país son pocos, no debemos restar importancia a la enfermedad; un solo caso merece nuestra atención, y nos debemos esforzar para encontrar el procedimiento eficaz y seguro en la estética de las mamas.

El reporte del primer caso en nuestro país se hizo en 2016 por el Dr. César Torres Rivero en el Estado de Querétaro; ${ }^{4}$ encontramos cuatro casos y después seis casos confirmados. El número sin duda seguirá creciendo conforme pase el tiempo, ya que tenemos mayor conocimiento sobre la enfermedad y son más los cirujanos que están convencidos de la importancia de su detección.

Cabe mencionar que, en la región de América Latina, en nuestro congreso anual en Cancún, realizamos un consenso para saber qué estaba pasando con esta enfermedad. ${ }^{5}$ Con los resultados obtenidos realizamos un protocolo para la detección de la enfermedad y su manejo.

Es importante mencionar que no en todos los países se reporta el mismo número de casos. A mayor cantidad de implantes colocados, mayor es la cantidad de casos reportados; además, existen otros factores intrínsecos de cada país, como los aspectos demográficos, genéticos, culturales y ambientales. Por ejemplo, en Estados Unidos de Norteamérica, que es el principal país donde se practican este tipo de procedimientos, hay un registro de 200 casos. En Europa, no todos los países reportan los mismos números; existe una mayor cantidad de reportes en Francia, con 55 casos; en Alemania o países de Europa central como Rumania, el número es menor. ${ }^{6}$ Son pocos los reportes en pacientes negras o asiáticas. En América Latina se tiene conocimiento de manera verbal de varios casos en diferentes países, sin embargo, no contamos con la publicación para tener mayor detalle de lo que pasa en nuestra región.

El linfoma no Hodgkin no es exclusivo de los implantes mamarios, pues existen reportes de linfoma en otras prótesis como cadera y rodilla; prótesis dentales o glúteo; procedimientos endovasculares e incluso en una banda de cirugía bariátrica. ${ }^{7-12}$ Aunque las características demográficas son distintas, en la mayoría de los casos se manifiesta el linfoma como un problema local, con una masa o enrojecimiento alrededor de la prótesis. En el caso de mama o glúteo, la enfermedad se presenta en la mayoría de los casos como un seroma crónico. Este último se define como todo aquel que se presenta en un tiempo mayor a un año después de la colocación de la prótesis.

A continuación, mencionaremos los puntos más relevantes del linfoma relacionado con los implantes mamarios BIA-ALCL, lo cual está orientado a la detección y tratamiento temprano.

\section{Presentación clínica del BIA-ALCL}

El linfoma asociado con implantes mamarios se denomina linfoma anaplásico de células gigantes BIA-ALCL, a diferencia del que se presenta en una mama sin prótesis. El comportamiento es distinto, ya que generalmente en el BIA-ALCL, al retirar la capsula y el implante, el pronóstico es favorable. La mayoría de los casos con BIA-ALCL se presentan con un crecimiento mamario unilateral ocasionado por un seroma crónico. En promedio, esta patología se presenta a los diez años de la colocación de la prótesis en el cuerpo, con un rango de 1 a 40 años, según se reporta en las diferentes series. ${ }^{3,4}$ El crecimiento de esta patología es bilateral menos frecuentemente; en un número menor de casos existen adenopatías y/o una masa palpable, y algunos casos son asintomáticos. Se recomienda que las pacientes con implantes mamarios se revisen una vez al año, independientemente del seguimiento rutinario que se utiliza en todas las mujeres para la detección del cáncer de mama.

Se cuenta con una clasificación de TNM para el BIA-ALCL (Tabla 1 y 2). Lo importante es hacer un diagnóstico precoz, sobre todo cuando la enfermedad se localiza en la cápsula o en el seroma, ya que el tratamiento es menos agresivo y el pronóstico es mejor. Cuando la enfermedad ya ha afectado los ganglios o hay enfermedad a distancia, se requiere un manejo más agresivo por parte de un equipo multidisciplinario y el pronóstico es sombrío. ${ }^{5}$

En todos los casos de pacientes con implantes mamarios que presenten un aumento 


\begin{tabular}{ll}
$\begin{array}{c}\text { Tabla 1: TNM para linfoma anaplásico de } \\
\text { células grandes (LACG) asociado con implantes } \\
\text { mamarios. }\end{array}$ \\
\multicolumn{2}{c}{ TNM } \\
\hline T1 & Seroma o dentro de la cápsula \\
T2 & Infiltración temprana de la cápsula \\
T3 & Agregados celulares o infiltración en todas \\
& las capas de la cápsula \\
T4 & Extensión más allá de la cápsula \\
N0 & No invasión de ganglios \\
N1 & Un ganglio regional \\
N2 & Múltiples ganglios \\
M0 & No hay metástasis a distancia \\
M1 & Extensión a otros órganos \\
\hline
\end{tabular}

y/o inflamación de una de sus mamas es importante el estudio del seroma y de la cápsula. En el cambio de implantes en condiciones rutinarias, se debe también realizar el estudio de la cápsula y del líquido. El envío de los especímenes a laboratorios especializados ha permitido detectar seis casos de linfoma y otras patologías como infecciones por micobacteria, reacción linfoproliferativa al silicón y metaplasia sinovial. Para confirmar el diagnóstico en el caso de seroma, además de los estudios de ultrasonido y resonancia magnética, es importante realizar una punción guiada preferentemente por ultrasonido para facilitar la punción/aspiración y evitar el daño del implante. Se debe avisar al patólogo para que estudie la muestra sin dejar pasar mucho tiempo. En caso de que la muestra no se revise el mismo día, se debe fijar con alcohol al 96\% en una proporción $50-50 \%$ para su estudio posterior. ${ }^{5} \mathrm{Si}$ a pesar de ello la muestra sale negativa y el problema persiste, se debe retirar la cápsula y enviarla a estudio; la cápsula se puede enviar en formol. Se debe notificar al patólogo sobre la posibilidad de linfoma, ya que se requieren mayores cortes e inmunomarcadores para identificar la patología.

Se recomienda discutir con la paciente la posibilidad de retirar los implantes. Si por algún motivo se colocan nuevamente, se debe utilizar una textura lisa y, en caso de confirmar el diagnóstico, se deben retirar los implantes. ${ }^{5}$
En los casos sospechosos, los estudios diagnósticos deberán ser guiados por un equipo multidisciplinario. El hematólogo es una pieza clave. ${ }^{4,5}$

El tratamiento quirúrgico está encaminado a hacer una capsulectomía bilateral con retiro de los implantes mamarios. Es muy importante la resección completa de la cápsula; es recomendable la extracción en una pieza y sin rupturas. No todas las cápsulas son fáciles de retirar, en especial, cuando la cápsula es gruesa se hace difícil su resección. Si existe una ruptura y escape del líquido del seroma en el lecho quirúrgi$\mathrm{co}$, se debe continuar con el procedimiento quirúrgico. ${ }^{4,5}$ Es importante informar a la paciente de la posibilidad de hacer una herida más grande que la herida original cuando se realice e implante.

No es recomendable bajo ninguna circunstancia, mientras la evidencia no demuestre lo contrario, retirar o cambiar los implantes mamarios de una textura a otra en pacientes asintomáticas. El cambio por otro implante con textura diferente no hace al procedimiento más seguro y con menos riesgo, además, hacerlo es agregar una mayor agresión. Existen pocos casos reportados de BIA-ALCL con implantes lisos y la mayoría de estos casos tuvieron un implante texturizado previo. Si se hace el simple cambio de implantes, es importante que el cirujano y la paciente comprendan la importancia de estudiar la cápsula.

El seroma se define como la acumulación líquida sin fibrinógeno u otro factor de coagulación que puede ser separado de la sangre coagulada. El seroma se diferencia

Tabla 2: Estadio según el TNM.

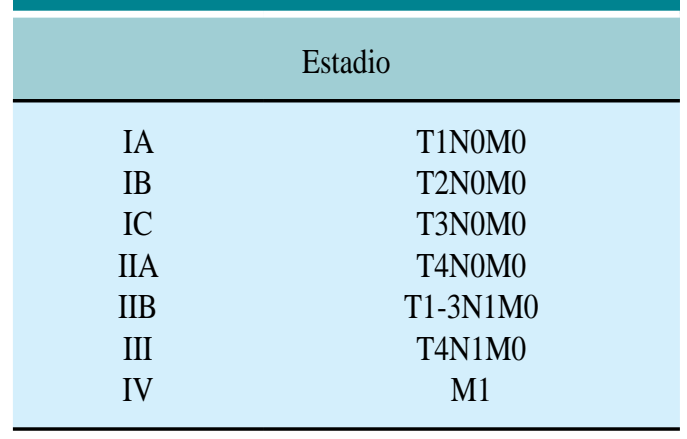


del plasma en que este último contiene líquido de sangre no coagulada en la que se encuentran suspendidas células hemáticas y tiene además factores de coagulación. ${ }^{13-15}$ En el examen del líquido, el seroma cuenta con acelularidad y pequeñas cantidades de proteínas menores a $2.0 \mathrm{~g} / \mathrm{dL}$, en comparación con un exudado, en el cual la cantidad es mayor a $2 \mathrm{~g} / \mathrm{dL}^{16}$

Se entiende al seroma, que fue definido por Tebbets, como aquel acúmulo de líquido corroborado por algún método de imagen con un volumen mayor a $20 \mathrm{~mL} .^{17,18}$ De no cumplir con estas características se definirá como líquido «periprotésico». ${ }^{19-21}$

En cuanto a la temporalidad, el seroma se puede clasificar como:

- Seroma temprano: $<6$ meses.

- Seroma intermedio: de 6 meses a 1 año.

- Seroma tardío o crónico: $>1$ año. ${ }^{22}$

En la literatura se reporta una incidencia entre 0.88 y $1.84 \%$, y hay diferentes teorías en cuanto a la fisiopatología, entre éstas destacan: ${ }^{22,23}$

1. Infección

2. Hematoma

3. Trauma

4. Enfermedades linfoproliferativas

5. Idiopático

6. Metaplasia sinovial

Infección: se ha encontrado bacterias grampositivas colonizando el bolsillo, secundarias a la contaminación de éste, al momento de su colocación. 24,25

Hematoma: la presencia de un hematoma no drenado provoca una irritación crónica que se puede manifestar con la aparición de seroma.

Trauma: un traumatismo puede evolucionar en un hematoma o bien sólo provocar un proceso inflamatorio que evolucionará a la formación de seroma. ${ }^{26-28}$

Metaplasia sinovial: ocasionada por el cizallamiento mecánico entre el implante y la cápsula, lo cual ocasiona un estado inflamatorio crónico con el desarrollo resultante del seroma. ${ }^{29,30}$
Se ha encontrado una mayor incidencia de seroma en pacientes con factores de riesgo como: obesidad, antecedente de radiación, diabetes mellitus y uso de matriz dérmica. ${ }^{31-33}$

La reconstrucción mamaria debe manejarse con otro apartado, ya que se han encontrado factores asociados propios de estos pacientes, tales como el estado hipovascular de los tejidos, aumento de espacio muerto, contaminación por biofilm, cuerpos extraños y disrupción linfática, que contribuyen al desarrollo de seroma, infección y pérdida protésica. ${ }^{34}$

Una vez que exista la sospecha clínica se deberá realizar un ultrasonido y, de ser posible, una punción guiada con toma de cultivo. El seroma crónico se deberá enviar a estudio de inmunohistoquímica para valorar la posibilidad de BIA-ALCL. Se deberá realizar la aspiración hasta obtener de 20 a $100 \mathrm{~mL}$ de líquido. Además del estudio citoquímico y citológico es importante realizar la tipificación histopatológica del mismo, buscando intencionadamente lo siguiente: ${ }^{5}$

- Cultivo para búsqueda intencionada de micobacteria.

- Conteo celular y citología para descartar datos sugestivos de ALCL.

- CD30 positivo

- ALK negativo

- Expresión de citoqueratina

- Traslocación $t^{5}$

Descartada la existencia de BIA-ALCL, se continuará su manejo como seroma crónico no asociado a ALCL. ${ }^{5}$ Existen varios factores de riesgo asociados al seroma, 19,20 para prevenirlo se recomienda cuidados generales pre y transoperatorios para evitar la contaminación y el biofilm, estos son (Tabla 3):

- Usar antibiótico intravenoso profiláctico al momento de la inducción.

- Evitar incisiones periareolares.

- Usar protectores de pezón.

- Realizar disección atraumática para minimizar devascularización.

- Hemostasia cuidadosa. 
- Usar bolsillos de plano dual.

- Emplear irrigación de bolsillo con triple antibiótico o solución de betadine ${ }^{\circledR}$ al $50 \%$.

- Usar un introductor plástico.

- Minimizar el tiempo de exposición del implante.

- Cambiar los guantes sin talco y asear las manos nuevamente.

- Evitar drenajes.

- Realizar cierre por planos.

- Usar profilaxis antibiótica en procedimientos subsecuentes que incidan piel o mucosa.

- Realizar el cierre de grandes bolsillos en casos de reconstrucción mamaria.

- Evitar actividad física intensa postoperatoria por 8 a 12 semanas.

Una vez instaurado el seroma, se debe tomar en cuenta el tiempo de evolución postquirúrgico. En caso de ser menor de un año, el protocolo terapéutico es realizar punción evacuatoria, una toma de cultivo con antibiograma y administrar antibioticoterapia de acuerdo con el resultado, además de vigilancia estrecha del paciente y colocación de dispositivos torácicos compresivos. Si no presenta resolución, es necesario efectuar exploración quirúrgica para realizar capsulectomía total, valoración transoperatoria del retiro de implantes versus el cambio de los mismos por algunos de superficie lisa; también se debe realizar irrigación del bolsillo mamario con triple antibiótico y colo- cación de drenajes. En caso de continuar sin resolución del seroma será necesario el retiro del implante definitivo.

En caso de presentar resolución del cuadro, si dentro de un periodo de vigilancia de un año después presenta recidiva, es necesario realizar una exploración quirúrgica con todo el protocolo ya mencionado anteriormente.

En caso de ser ALCL (-) es necesario realizar un manejo similar a un seroma de menos de un año postquirúrgico y continuar el flujograma de acuerdo con los resultados y la evolución del mismo (Figura 1).

Como anexo a este trabajo se agrega la infografía sobre el tema hecha por el Comité de Seguridad para orientar a los cirujanos plásticos, personal de salud y pacientes.

\section{CONCLUSIÓN}

El linfoma anaplásico de células gigantes asociado con implantes mamarios BIA-ALCL es una patología nueva. Tendremos mayor información al crear conciencia, en los cirujanos y pacientes, de la importancia del estudio del líquido periprotésico de las cápsulas que cubren los implantes. Por lo pronto, no se debe cambiar el implante en una paciente asintomática. En los casos de cambio rutinario de implantes se debe enviar el líquido y la cápsula para estudio de laboratorio y patología para descartar un linfoma.

Tabla 3: Medidas para disminuir el seroma en pacientes a las que se les coloca una prótesis mamaria.

Usar profilaxis antibiótica intravenosa en la inducción anestésica

Evitar incisiones periareolares cuando se presenta galactorrea

Usar protectores de pezón para prevenir diseminación bacteriana en el bolsillo

Realizar disección atraumática para minimizar desvascularización

Efectuar hemostasia cuidadosa

Evitar disección de parénquima mamario

Usar bolsillo en plano dual
Realizar irrigación de bolsillo con solución de triple antibiótico o solución de betadine ${ }^{\circledR}$

Usar bolsillo introductor

Minimizar tiempo de exposición de implante

Cambiar guantes quirúrgicos previamente al manejo del implante, así como instrumentos limpios Evitar uso de drenajes, ya que son una entrada potencial de bacterias

Usar cierre por planos

Realizar una profilaxis antibiótica para cubrir procedimientos posteriores que involucren piel o mucosa 


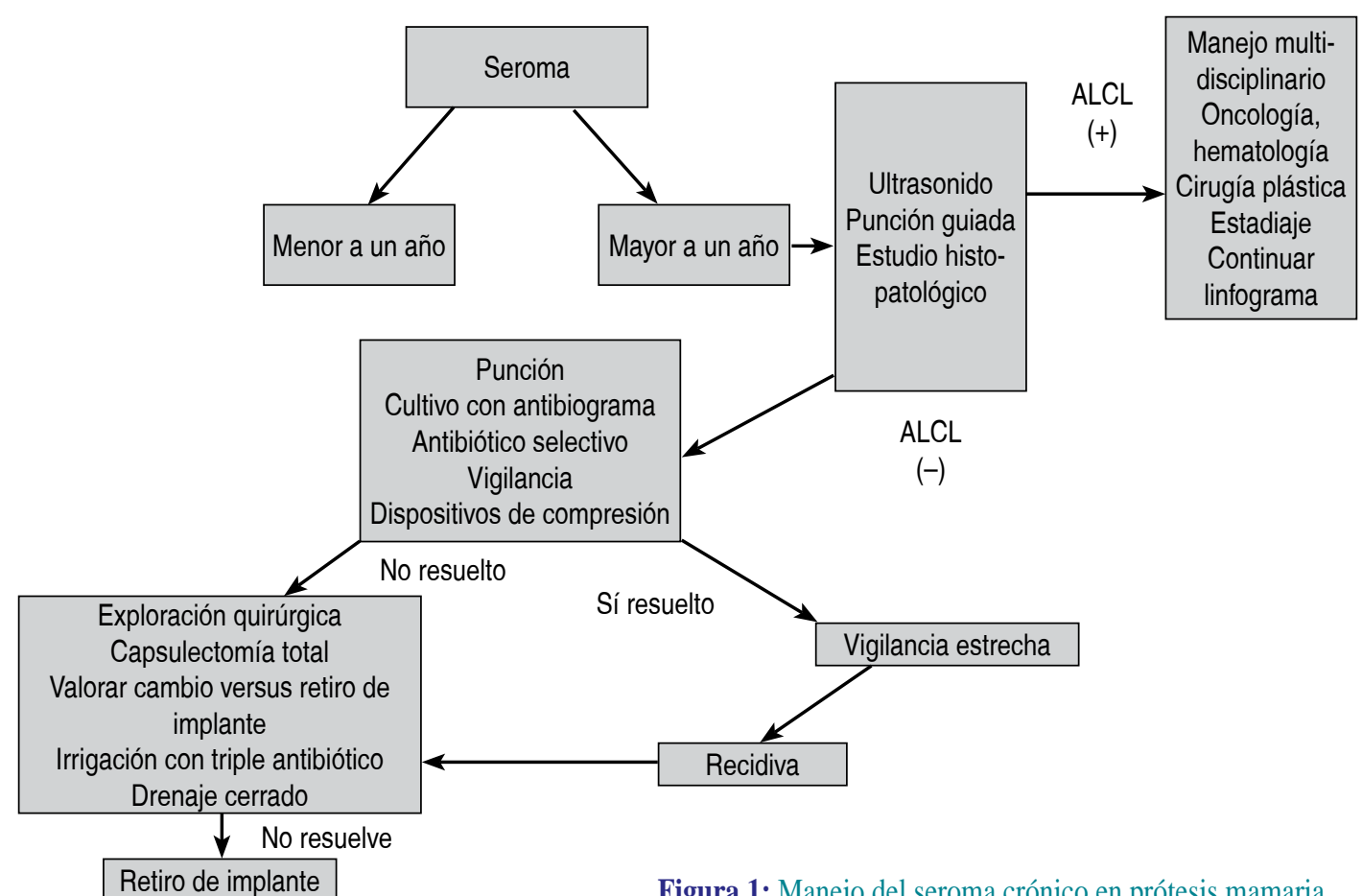

También se debe hacer la punción del seroma crónico guiada por ultrasonido para facilitar la punción/aspiración y para evitar una ruptura del implante.

Se debe avisar al patólogo en caso de tener una paciente a la cual se le va a puncionar un seroma crónico. Si por algún motivo el estudio no se puede hacer el mismo día de la punción, es recomendable usar alcohol al $96 \%$ en una proporción de 1 a 1 , esto es, $50 \%$ de muestra y $50 \%$ de alcohol para fijar la muestra.

Hay que informar a la paciente sobre el riesgo de linfoma y que éste quede registrado en el consentimiento informado.

Con la información actual podemos mencionar que en México la incidencia de BIA-ALCL es muy baja (0.000002\%: 2 casos en 1 millón). Si bien los números son distintos en otras partes del mundo, es necesario continuar un seguimiento en nuestras pacientes una vez al año para poder tener mayor información de esta enfermedad.

\section{REFERENCIAS}

1. Cook PD, Osborne BM, Connor RL et al. Follicular lymphoma adjacent to foreign body granulomatous inflammation and fibrosis surrounding silicone breast prosthesis. Am J Surg Pathol 1995; 19 (6): 712-717.

2. Keech JA Jr, Creech BJ. Anaplastic T-cell lymphoma in proximity to a saline-filled breast implant. PRS 1997; 100 (2): 554-555.

3. Ramos-Gallardo G, Cuenca-Pardo J, RodríguezOlivares E, Iribarren-Moreno R, Contreras-Bulnes L, Vallarta-Rodríguez $A$ et al. Breast implant and anaplastic large cell lymphoma meta-analysis. J Invest Surg 2017; 30 (1): 56-65.

4. Torres-Rivero C, Ramos-Gallardo G, Nambo-Lucio M, Vaquero-Pérez M. First case report in Mexico and Latin America of anaplastic large cell lymphoma in a patient with mammary implants. Cir Plast Iberolatinoam 2016; 42 (2): 175-180.

5. Ramos-Gallardo G, Cuenca-Pardo J, CardenasCamarena L, Duran-Vega H, Rodríguez-Olivares E, Bayter-Marin JE et al. Is Latin America Ready to Identify Anaplastic Large Cell Lymphoma in Breast Implants Patients? Regional Encounter during the National Plastic Surgery Meeting in Cancun, Mexico. Aesth Plast Surg 2018; 42 (5): 1421-1428.

6. Srinivasa D, Miranda RN, Kaura A, Francis AM, Campanale A, Boldrini R. Global Adverse Event Reports of Breast Implant Associated ALCL An International Review of 40 Government Authority Databases. PRS 2017; 139 (5): 1029-1039.

7. Sanchez-Gonzalez B, Garcia M, Ferran M, Sanchez M, Angona A, Salar AS. Diffuse Large B-Cell Lymphoma Associated with Chronic Inflammation In Metallic Implant. J Clin Oncol 2013; 31 (10): e148-e151.

8. Sunitsch S, Gilg M, Kashofer K, Leithner A, LieglAtzwanger B, Beham-Schmid C. Case Report: Epstein- 
Barr-Virus negative diffuse large B-cell lymphoma detected in a periprosthetic membrane. Diag Pathol 2016; 11 (1): 80-86.

9. Miller DV, Firchau DJ, McClure RF, Kurtin PJ, Feldman AL. Epstein-Barr Virus-associated Diffuse Large B-cell Lymphoma Arising on Cardiac Prostheses. Am J Surg Pathol 2010; 34 (3): 377-384.

10. Jin SH, Park G, Ko Y, Park JB. Diffuse Large B-Cell Lymphoma of the Peri-Implant Mucosa Mimicking Peri-Implants. J Oral Implantol 2016; 42 (2): 220-223.

11. Lee SH, Kim HC, Kim YJ. B-Cell Lymphoma in a Patient with a History of Foreign Body Injection. J Craniofac Surg 2017; 28 (2): 504-505.

12. Umakanthan MJ, McBride CL, Greiner Y, Yuan J, Sanmann J, Bierman PJ, Lunning MA, Bociek RG. Bariatric Implant-Associated Anaplastic Large-Cell Lymphoma. J Oncol Prac 2017; 13: 838-840.

13. Adams WP, Bengtson BP, Glicksman CA et al. Decision and management algorithms to address patient and food and drug administration concerns regarding breast augmentation and implants. Plast Reconstr Surg 2004; 114: 1252-1257.

14. Gabriel SE, Woods JE, O'Fallon WM, Beard CM, Kurland LT, Melton LJ III. Complications leading to surgery after breast implantation. N Engl J Med 1997; 336: 677-682.

15. Alobeid B, Sevilla DW, El-Tamer MB, Murty VV, Savage DG, Bhagat G. Aggressive presentation of breast implant-associated ALK-1 negative anaplastic large cell lymphoma with bilateral axillary lymph node involvement. Leuk Lymphoma 2009; 50: 831-833.

16. Pinchuk V, Tymofii O. Seroma as a late complication after breast augmentation. Aesth Plast Surg 2011; 35 (3): 303-14

17. Miranda RN, Lin L, Talwalkar SS, Manning JT, Medeiros LJ. Anaplastic large cell lymphoma involving the breast: A clinic pathologic study of 6 cases and review of the literature. Arch Pathol Lab Med 2009; 133: 1383-1390.

18. Chourmouzi D, Vryzas T, Drevelegas A. New spontaneous breast seroma 5 years after augmentation: A case report. Cases J 2009; 2: 7126.

19. Oliveira VM, Roveda Junior D, Lucas FB et al. Late seroma after breast augmentation with silicone prostheses: A case report. Breast J 2007; 13: 421-423.

20. de Jong D, Vasmel WL, de Boer JP et al. Anaplastic large-cell lymphoma in women with breast implants. JAMA 2008; 300: 2030-2035.

21. Do V, Shifrin DA, and Oostendorp L. Lymphoma of the breast capsule in a silicone implant-reconstructed patient. Am Surg 2010; 76: 1030-1031.

22. Domchek SM, Hecht JL, Fleming MD, Pinkus GS, Canellos GP. Lymphomas of the breast: Primary and secondary involvement. Cancer 2002; 94: 6-13.

23. Thompson PA, Lade S, Webster H, Ryan G, Prince HM. Effusion-associated anaplastic large cell lymphoma of the breast: Time for it to be defined as a distinct clinicopathological entity. Haematologica 2010; 95: 1977-1979.

24. Falini B, Martelli MP. Anaplastic large cell lymphoma: Changes in the World Health Organization classification and perspectives for targeted therapy. Haematologica 2009; 94: 897-900.

25. Falini B, Pileri S, Zinzani PL et al. ALK lymphoma: Clinicopathological findings and outcome. Blood 1999; 93: 2697-2706.

26. Li S, Lee AK. Silicone implant and primary breast ALK-1 negative anaplastic large cell lymphoma, fact or fiction. Int J Clin Exp Pathol 2010; 3: 117-127.

27. Farkash EA, Ferry JA, Harris NL et al. Rare lymphoid malignancies of the breast: A report of two cases illustrating potential diagnostic pitfalls. J Hematop 2009; 2: 237-244.

28. Fodor L, Moscona R. Late post-traumatic intracapsular seroma after breast augmentation. J Plast Reconstr Aesthet Surg 2009; 62: e609-e610.

29. Sahoo S, Rosen PP, Feddersen RM, Viswanatha DS, Clark DA, Chadburn A. Anaplastic large cell lymphoma arising in a silicone breast implant capsule: A case report and review of the literature. Arch Pathol Lab Med 2003; 127: e115-e118.

30. Gaudet G, Friedberg JW, Weng A, Pinkus GS, Freedman AS. Breast lymphoma associated with breast implants: Two case reports and a review of the literature. Leuk Lymphoma 2002; 43: 115-119.

31. Bengtson BP. Complications, reoperations, and revisions in breast augmentation. Clin Plast Surg 2009; 36: 139-156.

32. Hasham S, Akhtar S, Fourie LR. Persistent seroma following breast prosthesis explantation: A case report and review. Eur J Plast Surg 2006; 28: 490-493.

33. Newman MK, Zemmel NJ, Bandak AZ, Kaplan BJ. Primary breast lymphoma in a patient with silicone breast implants: A case report and review of the literature. J Plast Reconstr Aesthet Surg 2008; 61: 822-825.

34. Henriksen TF, Hölmich LR, Fryzek JP et al. Incidence and severity of short-term complications after breast augmentation: Results from a nationwide breast implant registry. Ann Plast Surg 2003; 51: 531-539.

\footnotetext{
Correspondencia:

Dr. Guillermo Ramos-Gallardo

Asociación Mexica de Cirugía Plástica,

Estética y Reconstructiva, A.C.

Flamencos núm. 74, esquina con Félix Parra,

Col. San José Insurgentes, 03900,

Alcaldía Benito Juárez, Ciudad de México.

E-mail: guiyermoramos@hotmail.com
} 


\section{RECOMENDACIONES \\ DE SEGURIDAD}

Linfoma anaplásico de células

gigantes relacionado con

implantes mamarios (BIA-ALCL)

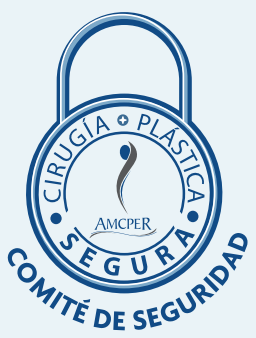

El número de casos ha ido aumentando, no se conoce la etiología, posiblemente relacionado a:

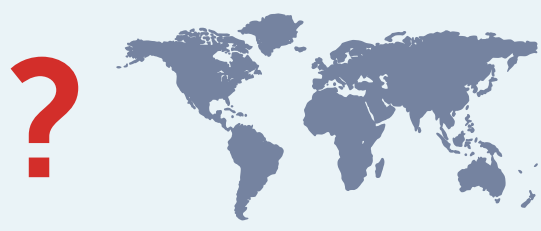

Posibles causas:

3. Relación a biofilm

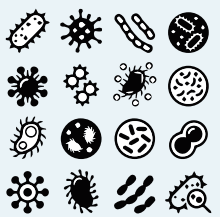

¿Cómo se presenta?
1. Inflamación crónica

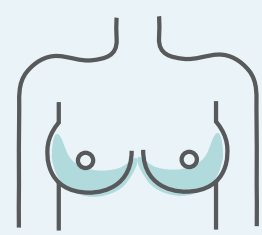

2. Genética

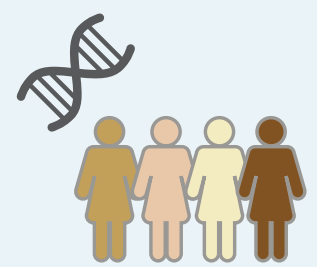

¿Cómo proceder ante un caso sospechoso?

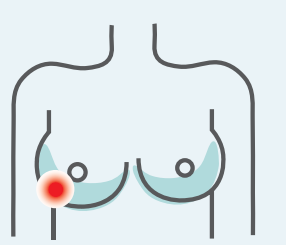

- Exploración física cuidadosa; buscar inflamación, seroma, masas tumorales, adenopatías

- Ultrasonido y resonancia magnética; buscar seroma, masas tumorales, adenopatías

- Extracción de seroma por punción y estudios de: citoquímicos, cultivos, patología

- Retiro de implantes, resección total de la cápsula de patología con inmunomarcadores

\section{Resultados}

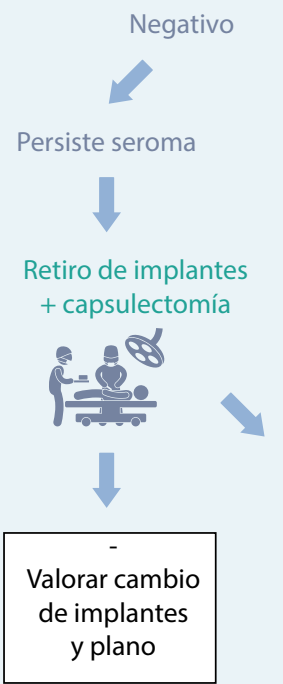

Positivo

Retiro de implantes + capsulectomía

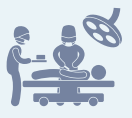

$+$

Tratamiento equipo multidisciplinario:

Oncólogo

Hematólogo

Cirujano Oncólogo

Radioncólogo
¿Qué hacer si detectamos un seroma?

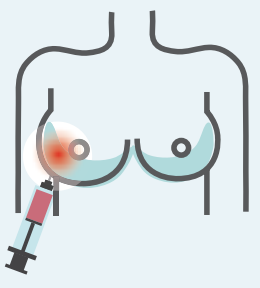

- Identificar el sitio del seroma; ultrasonido o resonancia magnética

- Paciente sentada

- Herida de $2 \mathrm{~mm}$. Introducir cánula punta roma y dar una descarga con el electrocauterio para puncionar la cápsula

- Extracción de la mayor cantidad de seroma

- Conservar el líquido en refrigeración y enviar inmediatamente al laboratorio de patología

\section{¿Cuáles son los estudios que debo solicitar?}

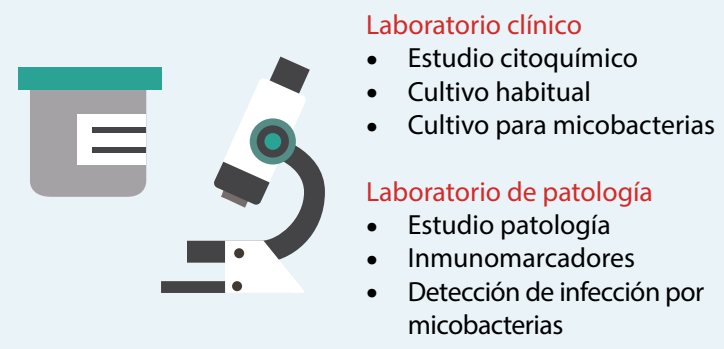

\section{Si cuentas con algún caso como}

éste, jcompártelo con nosotros!

Juntos podemos llegar a mejores resultados, detectar y manejar este tipo de patologías 